\title{
Realidad virtual como herramienta en fisioterapia, ¿ficción o realidad?
}

\author{
Virtual reality as a tool in physiotherapy: fiction or reality?
}

\author{
V. Robles García \\ Facultad de Fisioterapia, Universidad de A Coruña, Grupo de Neurociencia y Control Motor, A Coruña, España
}

En los últimos 20 años, la realidad virtual (RV) se ha incorporado en la práctica de la fisioterapia (principalmente neurológica) como una herramienta terapéutica que facilita la recuperación funcional. Pero, ¿qué aporta la RV a las intervenciones que realizamos? En realidad, para contestar a esta pregunta es importante remontarse a sus inicios y comprender así lo que el desarrollo de la tecnología de la RV nos ofrece en la actualidad.

Las primeras aplicaciones de la RV fueron en ámbitos tan diferentes al nuestro como el cine o la aeronáutica. «Ver es creer, pero sentir es estar seguro» es la frase que genéricamente podría encuadrar su concepción allá por los años 60, a manos de Morton Heilig, considerado el «padre» de la RV. Sin embargo, la concepción del término y el desarrollo de los sistemas de RV no han ido siempre de la mano, más bien, ha sido el desarrollo tecnológico el que ha marcado un antes y un después en sus posibilidades. Hoy en día, la RV se define como «un conjunto de tecnologías que permiten crear un entorno mediante la simulación de los estímulos sensoriales y la captura de los movimientos de los usuarios, favoreciendo así la interacción entre el entorno y el usuario de forma tal que este se sienta inmerso en el entorno virtual o incluso parte de él» ${ }^{1}$. De esta acepción se pueden destacar sus características principales ${ }^{2}$.

En primer lugar, los sistemas de RV se basan en tecnología que genera información de entrada al sistema (inputs) e información de salida (outputs) del sistema. Las acciones del usuario y sus movimientos son, por ejemplo, entradas que el sistema se encarga de capturar y presentar en el entorno virtual. En este momento, ya hay dispositivos que realizan un seguimiento preciso de los movimientos de las manos e incluso de los dedos. Por otro lado, las salidas del sistema pueden simular casi todas las modalidades sensoriales, pero se ha concedido mayor importancia a los estímulos visuales ya que son los que en un primer momento podían aportar mayor realidad a la escena virtual siendo, hoy en día, los inputs más desarrollados. Entre estos, son las gafas de RV oclusivas las que permiten más aislamiento del mundo exterior, y dentro de ellas, las mejores permiten el rastreo de la cabeza y/o de los ojos, cambiándose la escena virtual dependiendo de lo que mira el usuario ${ }^{3}$.

En segundo lugar, los sistemas han de cumplir la regla de las 3 «íes»: integración sensorial, interacción e inmersión. La tecnología RV más avanzada cuenta con sistemas de feedback sensorial multimodal. En ellos se emulan, por ejemplo, una imagen, un sonido, un olor o incluso la consistencia y el peso de un objeto. Como regla general, cuántas más vías sensoriales estimule el sistema de RV, más próxima a la realidad será la experiencia. Sin embargo, los sistemas de RV de bajo coste que solo emulan 
una o 2 modalidades sensoriales pueden conseguir niveles elevados de realismo siempre y cuando los estímulos presentados en el sistema y los que reciba del exterior sean congruentes entre sí (p. ej., escuchar el sonido de una puerta en el preciso momento que se cierra en el entorno virtual). Por otra parte, la interacción se refiere a la posibilidad de que el usuario perciba su propio movimiento dentro del entorno generado y que este tenga algún tipo de influencia en el mismo. En este sentido la coordinación entre los inputs y los outputs es esencial para que se produzca una acción recíproca entre el sistema y el usuario.

Cuanta más integración sensorial e interacción se permitan, mayor será el tercer punto, la inmersión. La inmersión se refiere a la capacidad de un dispositivo para generar presencia, es decir, que el usuario se sienta parte del entorno virtual ${ }^{4}$. El avatar o representación del usuario dentro del sistema ha de presentarse, idealmente, en perspectiva de primera persona (p. ej., viendo las manos como si fueran de uno) ya que se conoce que facilita la creación de ilusiones visuales mediante correlaciones visuotáctiles (parecido a la ilusión de la mano de goma reproducida en un experimento de RV en el laboratorio del Dr. Slater $^{5}$ ) o visuomotoras (gracias a las cuales se identifica el movimiento virtual como propio). Además, numerosos experimentos relacionados con el rol de las neuronas espejo (NE) durante el aprendizaje motor por observación e imitación de la acción — también en RV-, demuestran una mayor activación cortical si los movimientos son observados en primera persona más que en tercera, tanto si esta última se realiza en visión especular (p. ej., moviendo justo la mano correspondiente a la que se observa, izquierdaderecha) como anatómica (p. ej., moviendo la misma mano, derecha-derecha) ${ }^{6}$.

Ciertamente, nos interesan los tipos y características de los sistemas de RV porque, en gran medida, influyen en los efectos que podamos conseguir, dependiendo de cómo y para qué se utilicen en nuestra intervención fisioterapéutica. El ejemplo más claro se remonta a los inicios del uso clínico de los sistemas de RV en el tratamiento del miedo a las alturas (o acrofobia). Después de desarrollar el sistema, lo primero que hicieron los investigadores fue comprobar que las escenas virtuales generaban la misma respuesta de ansiedad y miedo en los pacientes que las situaciones reales. Y esto lo consiguieron, pero con 2 ventajas a mayores, por un lado, mejorar la exposición gradual al estímulo aversivo, y por el otro, realizarlo en un entorno seguro. Además, siendo el entorno virtual tan parecido al real se aseguraban de que hubiera una mayor transferencia de los resultados obtenidos, a partir de una actividad en el entorno virtual, a una situación real.

Además, ciertos sistemas de RV permiten medir, controlar e incluso modificar no solo los estímulos sensoriales presentados sino también las interacciones realizadas por parte del usuario. Medir las variables que registra el sistema nos permite realizar una cuantificación objetiva en las evaluaciones iniciales y finales, así como conocer la progresión del proceso terapéutico, por lo que es una herramienta ideal para establecer los objetivos y reevaluarlos al cabo de un tiempo junto al paciente. Controlar el entorno virtual nos permite regular el número, la frecuencia y la variedad de los estímulos o de la actividad que se lleva a cabo, así como individualizar la sesión, optimizando la intensidad de la intervención de acuerdo, por ejemplo, a la fatiga o al procesamiento cognitivo requerido.

Y, por último, la capacidad de modificar lo que pasa en el entorno virtual, es quizá la cualidad que le confiere más ventajas. La mayoría de los sistemas de RV permite escenarios hipotéticos (p. ej., una playa o un partido de football) que claramente incrementan la motivación del usuario y la adherencia al tratamiento (sobre todo en niños). También, en esta línea, se ha descrito como la representación virtual de un miembro que no existe en la realidad, como ocurre en el síndrome del miembro fantasma, disminuye los síntomas de dolor y sensibilidad que tienen aumentados estos pacientes. Por otro lado, al permitir modificar la representación virtual del movimiento realizado por el usuario, se pueden generar correlaciones visuomotoras (p. ej., imitando al avatar) que, después de un periodo de intervención, mejoren el movimiento real de los pacientes. Esto lo vimos en diferentes estudios en los que se investigó acerca de los efectos de un sistema de RV con voluntarios con enfermedad de Parkinson ${ }^{7,8}$.

En la enfermedad de Parkinson, suelen producirse signos motores como la bradicinesia, el temblor, la hipocinesia y la arritmocinesis. Se conoce que estos signos afectan a los movimientos repetitivos tanto en miembros superiores como inferiores, impactando visiblemente a la marcha. En un modelo básico de movimiento repetitivo, el golpeo repetitivo del dedo índice, observamos que la modificación de ciertos 
parámetros del movimiento del dedo del avatar (amplitud, cadencia, etc.) respecto a la ejecución real del paciente, permitía a este «corregir» su patrón motor al realizar el movimiento, mejorando así el reclutamiento muscular y disminuyendo la arritmocinesis del mismo mientras practicaba observando la escena virtual en un sistema RV (sistema que desarrollamos y validamos al efecto).

Tras estos resultados, se llevó a cabo una intervención terapéutica con el fin de conocer si los efectos observados en una sola sesión se optimizaban y mantenían tras un período de imitación de un movimiento corregido realizado por el avatar. Al cabo de 4 semanas, los resultados obtenidos demostraron una mejora de la amplitud de movimiento solo en este grupo (grupo experimental), que además se transfería al entorno real y que persistía después de 2 semanas de seguimiento. Sin embargo, en el grupo control, que practicaba en el mismo sistema de RV, pero viendo su propio movimiento en el entorno virtual (sin modificar), no se producía esta mejoría, demostrando así que la posibilidad de modificar el movimiento en el entorno virtual es una característica que genera ventajas en los efectos conseguidos.

En esta línea y en general, los estudios que versan sobre la aplicación de la RV muestran sus efectos en la recuperación funcional de usuarios con enfermedad neurológica. Así, se han demostrado mejorías en el equilibrio, la marcha y la funcionalidad de miembro superior en pacientes con esclerosis múltiple ${ }^{9}$, Parkinson y con secuelas tras haber sufrido un $\mathrm{ACV}^{10}$. También, existen estudios (pocos) acerca de su utilización para la «distracción» del dolor y, cada día más, en el ámbito del deporte.

Por otro lado, la mayor parte de la literatura que estudia los efectos de la RV en el sistema nervioso central describe cambios en los circuitos corticales (y con ellos, también en el rendimiento motor) siempre y cuando se presentan estímulos virtuales apropiados con el fin de promover la adaptación de los patrones motores de los usuarios ${ }^{11}$. Esta adaptación puede producirse por los diferentes elementos de los sistemas de RV: los más sencillos, mediante feedback sensorial y los sistemas más completos o más inmersivos, facilitando el aprendizaje basado en la práctica en un entorno motivador o el aprendizaje basado en la observación de la acción y/o imitación en un entorno modificado (siendo esta última la que más cambios ha demostrado en la activación de redes neuronales).

En conclusión, las características del entorno virtual son críticas para comprender las repercusiones que tiene este sobre el sistema nerviosos central, y por ende, sobre la recuperación funcional que esperamos obtener en nuestros pacientes. Se acerca, si no ha comenzado ya, una época en la que la tecnología más inmersiva está a nuestro alcance. Y en la aplicación de esta con fines terapéuticos, tenemos el rol de emplearla meticulosamente para rendir su máximo potencial: la posibilidad de emular un movimiento incluyendo (casi) todas sus connotaciones sensoriales.

\section{Bibliografía}

1. Sherman WR, Craig AB. Understanding Virtual Reality: Interface, Application, and Design. Amsterdam: Morgan Kaufmann Publishers; 2003.

2. Cudeiro Mazaira J, Arias Rodríguez P, Mariño Alfonso X, et al. Reeducación funcional en la enfermedad de parkinson. 2.a ed. Barcelona: Elsevier; 2014.

3. Kim A, Darakjian N, Finley JM. Walking in fully immersive virtual environments: An evaluation of potential adverse effects in older adults and individuals with Parkinson's disease. J Neuroeng Rehabil. 2017;14:16, http://dx.doi.org/10.1186/s12984-017-0225-2 .

4. Sanchez-Vives MV, Slater M. From presence to consciousness through virtual reality. Nat Rev Neurosci. 2005;6:332-9, http://dx.doi.org/10.1038/nrn1651 .

5. Slater M, Perez-Marcos D, Ehrsson HH, Sanchez-Vives MV. Inducing illusory ownership of a virtual body. Front Neurosci. 2009;3:214-20, http://dx.doi.org/10.3389/neuro. 01.029.2009 .

6. Jackson PL, Meltzoff AN, Decety J. Neural circuits involved inimitation and perspective-taking. Neuroimage. 2006;31:429-39, http://dx.doi.org/10.1016/j.neuroimage. 2005.11.026.

7. Robles-García V, Arias P, Sanmartín G, Espinosa N, Flores J, Grieve KL, et al. Motor facilitation during real- time movement imitation in Parkinson's disease: A virtual reality study. Parkinsonism Relat Disord. 2013;19:1123-9, http://dx.doi.org/10.1016/j.parkreldis.2013.08.005. 
8. Robles-García V, Corral-Bergantiños Y, Espinosa N, García- Sancho C, Sanmartín G, Flores J, et al. Effects of movement imitation training in Parkinson's disease: A virtual reality pilot study. Parkinsonism Relat Disord. 2016;26:17-23, http://dx.doi.org/10.1016/j.parkreldis.2016.02.022

9. Peruzzi A, Zarbo IR, Cereatti A, Della Croce U, Mirelman A. An innovative training program based on virtual reality and treadmill: Effects on gait of persons with multiple sclerosis. Disabil Rehabil. 2017;39:1557-63, http://dx.doi.org/10.1080/09638288.2016.1224935.

10. de Rooij IJM, van de Port IGL, Meijer JWG. Effect of virtual reality training on balance and gait ability in patients with stroke: Systematic review and meta-analysis. Phys Ther. 2016;96:1905-18, http://dx.doi.org/10.2522/ptj.20160054.

11. Adamovich SV, August K, Merians A, Tunik E. A virtual reality-based system integrated with fmri to study neural mechanisms of action observation-execution: A proof of concept study. Restor Neurol Neurosci. 2009;27:209-23, http://dx.doi.org/10.3233/RNN-2009-0471 . 